\title{
Validez e invariancia factorial del Índice de Bienestar General (WHO-5 wBI) en universitarios peruanos
}

\author{
Validity and Factorial Invariance of the General \\ Well-Being Index (wHo-5 wBI) \\ in Peruvian University Students
}

Validade e invariância fatorial do Índice Geral de Bem-Estar (WHO-5 wBI) em universitários peruanos

Tomás Caycho-Rodríguez ${ }^{1 *}$

José Ventura-León ${ }^{1,2}$

Karla Azabache-Alvarado 1,3

Mario Reyes-Bossio 4

Isabel Cabrera-Orosco ${ }^{5}$

Recibido: 4 de febrero de 2020 • Aceptado: 8 de julio de 2020

Doi: https://doi.org/10.12804/revistas.urosario.edu.co/revsalud/a.9797

Para citar este artículo: Caycho-Rodríguez T, Ventura-León J, Azabache-Alvarado K, Reyes-Bossio M, Cabrera-Orosco I. Validez e invariancia factorial del Índice de Bienestar General (wHo-5 wBI) en universitarios peruanos. Rev Cienc Salud. 2020;18(3):1-23. https:// doi.org/10.12804/revistas.urosario.edu.co/revsalud/a.9797

\section{Resumen}

Introducción: actualmente, existe un mayor interés por el estudio del bienestar, debido a su importancia en el desarrollo y evaluación de políticas en salud a favor de la población en general. Por lo tanto, se hace necesaria una medición válida y confiable del bienestar. En este contexto, el estudio evaluó las evidencias de validez basadas en la estructura interna, confiabilidad e invarianza factorial por sexo del índice de Bienestar General (wHo-5 wBI). Materiales y métodos: participaron 499 estudiantes universitarios de la ciudad de Trujillo (Perú) de sexo femenino $(n=271)$ y masculino $(n=228)$. Se realizó un análisis factorial

1 Universidad Privada del Norte (Perú).

Autor de correspondencia:tomas.caycho@upn.pe. oRciD: https://orcid.org/0000-0002-5349-7570

2 ORCID: https://orcid.org/0000-0003-2996-4244

3 ORCID: https://orcid.org/0000-0003-3567-4606

4 Universidad Peruana de Ciencias Aplicadas (Perú). orCiD: https://orcid.org/0000-0003-4655-1927

5 Universidad Continental (Lima). oRCID: https://orcid.org/0000-0002-0375-2879 
confirmatorio, confiabilidad por consistencia interna e invarianza factorial por sexo mediante un análisis multigrupo. Resultados: el análisis factorial confirmatorio indicó que el modelo unidimensional de cinco ítems con errores correlacionados de los ítems 1 y 4 presentaba un buen ajuste $\left(\chi^{2}=9.667\right.$ [gl = 4]; $\mathrm{p}>0.05 ; \chi^{2} / \mathrm{gl}=2.416 ;$ сFI $=0.994 ;$ RMSEA $=0.053$ [Iс90\%: 0.006-0.097]; SRMR $=0.018$ ). Además, se reportó una confiabilidad aceptable $(\omega=0.758)$ e invariancia factorial por sexo. Conclusión: el wHo-5 WBI demostró evidencia empírica a favor de la validez basada en la estructura interna, confiabilidad de las puntuaciones e invariancia factorial, que permiten contar con un instrumento útil y rápido para la medición del bienestar subjetivo en estudiantes universitarios.

Palabras clave: bienestar subjetivo; confiabilidad; invarianza factorial; validez.

\section{Abstract}

Introduction: Owing to its importance in the development and evaluation of health policies in favor of the general population, there is currently a greater interest in the study of well-being. Therefore, a valid and reliable measurement of well-being is necessary. In this context, this study evaluated the evidence of validity based on the internal structure, reliability, and factor invariance by sex of the General WellBeing Index (wнo-5 wвI). Materials and Methods: A total of 499 university students (female $=271$; male = 228) from the city of Trujillo, Peru, participated in the study. A confirmatory factor analysis, reliability by internal consistency, and factor invariance by sex were performed using a multigroup analysis. Results: The confirmatory factor analysis indicated that the 5-dimensional model with 5 correlated errors of items 1 and 4 presented a good fit $\left(\chi^{2}=9.667[\mathrm{gl}=4], \mathrm{p}>0.05 ; \chi^{2} / \mathrm{gl}=2.416 ; \mathrm{CFI}=0.994 ;\right.$ RMSEA $=0.053$ [IC90\% 0.006-0.097]; SRMR $=0.018)$. In addition, acceptable reliability $(\omega=0.758)$ and factor invariance by sex were reported. Conclusion: The wно- 5 wBI demonstrated empirical evidence in favor of validity based on internal structure, reliability of scores, and factor invariance that allow for a useful and rapid instrument for measuring subjective well-being in university students.

Keywords: Factorial invariance; reliability; subjective well-being; validity.

\section{Resumo}

Introdução: atualmente existe um maior interesse pelo estudo do bem-estar, devido à sua importância no desenvolvimento e avaliação de políticas em saúde a favor da população em geral. Portanto, se faz necessária uma medição válida e confiável do bem-estar. Neste contexto, o estudo avaliou as evidências de validade baseada na estrutura interna, confiabilidade e invariância fatorial por sexo do Índice Geral de Bem-Estar (wно-5 wвI). Materiais e métodos: participaram 499 estudantes universitários da cidade de Trujillo (Peru) de sexo feminino $(n=271)$ e masculino $(n=228)$. Se realizou uma análise fatorial confirmatória, confiabilidade por consistência interna e invariância fatorial por sexo mediante uma análise multigrupo. Resultados: a análise fatorial confirmatória indicou que o modelo unidimensional de cinco itens com erros correlacionados dos itens 1 e 4, apresentava um bom ajuste $\left(\chi^{2}=9,667\right.$ [gl $\left.=4\right], p>0,05$; $\chi^{2} / \mathrm{gl}=2,416$; $\mathrm{CFI}=0,994 ;$ RMSEA $=0,053$ [IC90\% 0,006-0,097]; SRMR = 0,018). Além disso, se reportou uma confiabilidade aceitável $(\omega=0,758)$ e invariância fatorial por sexo. Conclusão: se conclui que o wHo-5 wBI demostrou evidência empírica a favor da validade baseada na estrutura interna, confiabilidade das pontuações e invariância fatorial que permitem contar com um instrumento útil e rápido para a medição do bem-estar subjetivo em estudantes universitários.

Palavras-chave: bem-estar subjetivo; confiabilidade; invariância fatorial; validade. 


\section{Introducción}

$F^{n}$ los últimos años ha existido un mayor interés en el mundo por los estudios acerca del lineamientos actuales de la Organización Mundial de la Salud (oms), que sugieren adoptar una perspectiva positiva de la salud que permita a las personas identificar sus potencialidades, trabajar de manera productiva, enfrentar los problemas cotidianos de la vida y contribuir al desarrollo de su comunidad (2).

Tradicionalmente, las investigaciones sobre el bienestar han discurrido en función de dos tradiciones teóricas: una postura eudemónica, orientada al estudio del bienestar psicológico, y una visión hedónica, centrada en el bienestar subjetivo (BS)(1). El BS se define como el grado de bienestar experimentado por cada persona de acuerdo con una evaluación subjetiva de su vida, que incluye un conjunto de juicios cognitivos y reacciones afectivas, según las experiencias previas, el estado de vida presente y las expectativas (3). El Bs está conformado por dos dimensiones relacionadas: una cognitiva, representada por la satisfacción con la vida, y otra emocional, expresada en los afectos positivos y afectos negativos (4).

$\mathrm{El}$ Bs es un tema de interés en diferentes contextos, entre ellos el universitario (5), debido a su relación con el éxito académico y las metas profesionales (6). La evidencia en Latinoamérica señala que la población universitaria presenta índices altos de Bs, lo que muestra una tendencia a experimentar mayores afectos positivos que negativos, relacionados con la autoeficacia, el optimismo y el rendimiento académico (7-9). Asimismo, los sentimientos que tienen las personas acerca de sí mismas son un adecuado predictor del desgaste, salario y tipo de trabajo futuro del universitario (10). En este sentido, las relaciones estudiantiles y el apoyo social son importantes para el mantenimiento de una elevada motivación académica que, a su vez, facilitaría el bienestar de los universitarios (11). Además de lo anterior, el estudio del Bs es importante para desarrollar y evaluar políticas en salud a favor de la población en general (12). Por ello, en los últimos años, países como Canadá están considerando los índices de Bs con los fines mencionados (13).

En la literatura sobre el tema se observan diversos instrumentos para medir el Bs general o aspectos particulares que, a su vez, se han desarrollado para una población general o específica (14). Entre los instrumentos, el Índice de Bienestar General (wHo-5 wBI, por sus siglas en inglés) es una escala autoadministrada breve (cinco ítems) y genérica para evaluar el Bs durante los últimos 14 días (15). Los ítems del wHo-5 wBI reflejan la presencia de buen humor, sensación relajada, vitalidad e interés por las cosas. A pesar de su sentido positivo, el wHo-5 wBI puede emplearse también como una medida corta y rápida con el propósito de detectar la depresión en países con diferentes recursos económicos (16). La medición del bienestar, en lugar de los síntomas depresivos, es un procedimiento más aceptado por los pacientes y eficaz para detectar depresión. Así, una falta de bienestar sería 
un indicador de la existencia de un cuadro depresivo (17). Además, es posible usar el who-5 WBI en encuestas nacionales, ya que sus ítems no tienen en cuenta las características únicas de una población específica. Los orígenes del wHo-5 wBI se encuentran en el proyecto sobre calidad de la atención en pacientes con diabetes insulino-dependiente, desarrollada por la Oficina Regional de la oms para Europa. La escala inicial tuvo 28 ítems, la que derivó luego en la versión de cinco ítems, que utilizamos en este estudio y que están formulados solo de manera positiva (18). Desde entonces, el wHo-5 wBI se ha traducido a más de treinta idiomas (véase https:// www.who-5.org) (16) y administrado a diversas muestras como estudiantes adolescentes (19), adultos mayores (20) y en diferentes condiciones de salud, como trastornos depresivos (17) y pacientes eutímicos con trastorno bipolar (21).

En relación con sus propiedades psicométricas, diversos estudios reportan la existencia de un solo factor del who-5 wBI que explica entre el $56.7 \%$ y el $70.8 \%$ de la varianza total y una adecuada consistencia interna, con coeficientes alfa de Cronbach entre 0.66 y 0.95 (19-26). Una revisión sistemática demostró que los cinco ítems del wHo-5 wві conforman una medida unidimensional en que cada uno ellos brindan una información única acerca del bienestar. De igual manera, al analizar la totalidad de estudios para detectar depresión, se observaron índices de sensibilidad y especificidad de 0.86 y 0.81 , respectivamente, considerados aceptables (16).

A pesar de que el who-5 wвI es una de las medidas cortas más utilizadas, sobre todo en disciplinas médicas, se carece de una versión validada para el Perú. Además, las propiedades psicométricas del wHo-5 wBI en estudiantes universitarios no se han analizado en el contexto latinoamericano, sino en Iraq y Rumania $(27,28)$. Estos estudios solo han evaluado la estructura del wHo-5 wBI con base en el análisis factorial exploratorio y la correlación de su puntaje total con otras variables teóricamente asociadas. Por otro lado, no existen estudios en el contexto universitario que hayan probado la invariancia factorial de los ítems del шно-5 шві en diferentes subgrupos, como el sexo. Solo algunas investigaciones han evaluado la invarianza del wHo-5 wвI en muestras diferentes a los estudiantes universitarios. Estos resultados indican que la estructura unidimensional es invariante entre personas deprimidas y no deprimidas, así como entre hombres y mujeres de la población en general $(23,26)$. La invarianza factorial brinda evidencia empírica acerca de la estabilidad de la estructura del instrumento de medida entre diferentes grupos, por lo que es un requisito en aquellas investigaciones que comparan grupos (29).

La ausencia de invarianza factorial aumenta las probabilidades de sesgo en la medición a favor de uno de los grupos comparados, debido a la presencia de variables extrañas, lo que afecta la validez de los resultados (29). Asimismo, la brevedad y la facilidad de administración del wно-5 wBI, junto con la facilidad para detectar cambios en el bs y no ser una medida específica para casos de enfermedad, también justifican su estudio psicométrico (19). En este sentido, el propósito del presente estudio fue analizar las evidencias iniciales de validez basada en la estructura interna, 
la confiabilidad y la invarianza factorial entre hombres y mujeres del wHo-5 wBI en población universitaria de un país latinoamericano, como el Perú. Con base en la evidencia empírica, se planteó como hipótesis que el modelo de un factor resulta en un buen ajuste. Además, se espera una fiabilidad aceptable y que la estructura factorial será invariante en función sexo.

\section{Materiales y métodos}

$F^{l}$ diseño del estudio fue instrumental, debido a que se evaluaron las propiedades psidos universidades (pública y privada) de la ciudad de Trujillo (Perú), seleccionados por un muestreo intencional y que cumplían con los siguientes criterios de inclusión: a) tener 18 años o más, b) estar matriculados en la universidad seleccionada durante el estudio y c) consentir libremente su participación. Por otro lado, se consideraron criterios de exclusión: a) participantes con problemas físicos graves o discapacidad aparente, b) individuos que autoinformaron haber sido diagnosticados con algún problema de salud mental y estuvieron o están bajo tratamiento psiquiátrico durante el año anterior a la investigación y c) aquellos que no lograron completar el cuestionario. En el análisis de invarianza factorial se procuró que fueran similares los tamaños muestrales de hombres y mujeres. El cálculo del tamaño muestral (n) siguió la recomendación de Schumacker y Lomax, quienes sugieren un $n$ entre 100 y 500 participantes para estudios de este tipo (31).

En este sentido, participaron 499 estudiantes universitarios con una edad promedio de 20.71 años ( $\mathrm{DE}=2.61$ ), de los cuales 228 fueron hombres ( $45.69 \%$ ) y 271 mujeres $(54.31 \%$ ). Los hombres y mujeres tuvieron una edad promedio de 20.8 años $(D E=2,74)$ y 20.6 años $(\mathrm{DE}=2.50)$, respectivamente. La comparación de medias no evidenció diferencias significativas en el promedio de edad en función del sexo $\left(\mathrm{t}_{(497)}=0.884 ; \mathrm{p}=0.337 ; \mathrm{d}=0.08\right)$.

Como instrumento de medida se utilizó la versión en español del wHo-5 wBI, realizada por Simancas-Pallares et al., que es una medida de detección breve y efectiva del bienestar (25). Esta versión muestra una adecuada consistencia interna ( $\alpha=0.852 ; \omega=0.877)$ y una estructura factorial que explica el $56.17 \%$ de la varianza total con buenos índices de ajuste. Está conformado por cinco ítems (por ejemplo: "Me he sentido alegre y de buen ánimo") con 4 alternativas de respuesta tipo Likert ( $0=$ nunca, 1 = a veces, $2=$ muchas veces y $3=$ siempre). El puntaje total se obtiene con la sumatoria simple de los puntajes de cada ítem, donde 0 expresa una ausencia de bienestar y 15 un alto bienestar.

El estudio contó con el aval del Comité de Ética de la Universidad Privada del Norte, a la cual está suscrito el primer autor y que fue la institución que financió el proyecto. Se solicitaron los permisos a las autoridades de las instituciones universitarias públicas y privadas para la aplicación del wHo-5 wBI, a la vez que se informó acerca de sus características y el objetivo 
de la investigación. Una vez revisados los aspectos éticos y metodológicos, se obtuvo el aval respectivo. Enseguida, se procedió a informar a los estudiantes el objetivo del estudio, y por medio de la firma del consentimiento informado se obtuvo su participación voluntaria. Se aplicó el instrumento de manera colectiva en sus aulas de clases.

El análisis estadístico se llevó a cabo con el programa de acceso libre R, versión 3.1.2. Primero, se realizó un análisis factorial confirmatorio. No se efectuó un Análisis Factorial Exploratorio, porque teóricamente la escala asume la unidimensionalidad. Se siguió la clasificación de Mueller y Hancock, utilizando el estadístico chi-cuadrado $\left(\chi^{2}\right)$, el chi-cuadrado relativo ( $\left.\chi^{2} / g l\right)$, el error cuadrático medio de aproximación (RMSEA), la raíz residual estandarizada cuadrática media (SRMr) y el índice de ajuste comparativo (CFI) (32). Adicionalmente, se calculó el criterio de Akaike (AIC). Valores de $\chi^{2} / \mathrm{gl}<3$; CFI $>0.95$ y RMSEA y SRMR $<0.08$ permitieron considerar un buen ajuste del modelo (33). Con el fin de identificar posibles errores de especificación, se analizaron losíndices de modificación, donde valores significativos del $\chi^{2}$ se encuentran asociados con magnitudes mayores a 0.20 del Unstandardized Expected Parameter Change (34). En primer lugar, se evaluó el modelo unidimensional original, para luego incluir el modelamiento de errores correlacionados indicados por los índices de modificación como significativos.

Con base en la evidencia proporcionada mediante el análisis factorial confirmatorio se procedió a estudiar la if de la escala. Por tal motivo, se evaluó el modelo progresivamente, desde la invarianza configural (M1), relacionado con una estructura factorial sin restricciones; invarianza métrica (M2), donde se imponen restricciones en las cargas factoriales; invarianza fuerte (M3), donde las cargas factoriales e interceptos son idénticos e invarianza estricta (M4), que asume igualdad en cargas factoriales, interceptos, covarianza y varianza del error (29). Se calcularon los siguientes criterios de invariancia: la diferencia del chi-cuadrada $\left(\Delta \chi^{2}\right.$; $p>0.05)$ y $\Delta$ CFI $(\Delta$ CFI $<0.01)$ (35). Finalmente, se calcularon las diferencias de medias latentes entre hombres y mujeres a través de la $t$ de Student y el tamaño del efecto según lo sugerido por Cohen (36), donde $d=0.20$ es pequeño, $d=0.50$ es mediano y $d=0.80$ es largo.

Se estimó la confiabilidad mediante el coeficiente $\omega$, debido a que se utilizó un modelo de análisis factorial (37). Como uno de los modelos contó con errores correlacionados se calculó el $\omega$ con corrección para este caso $\left(\omega_{\text {corregido }}\right)$, donde son adecuados valores $\omega_{\text {corregido }}>0.80$ (38).

\section{Resultados}

T a hipótesis 1, que planteó que un modelo de un factor resulta en un buen ajuste, se sustentó ـpor los siguientes resultados. Primero, se evaluó el modelo original de un solo factor con los cinco ítems, cuyos índices de ajuste no fueron del todo aceptables $\left(\chi^{2}=36.022[\mathrm{gl}=5] ; \mathrm{p}<\right.$ 0.05; $\chi^{2} / \mathrm{gl}=7.204 ; \mathrm{CFI}=0.966 ;$ RMSEA $=0.112$ [IC 90\%: 0.079-0.147]; SRMR $=0.032$ ). Asimismo, se identificó una especificación cuyo Expected Parameter Change fue mayor a 0.20 entre 
el residual de los ítems 1 y 4, lo que sugiere una disminución significativa del valor $\chi^{2}$. Segundo, se incluyó la correlación de errores identificada, lo que mejoró los índices de ajuste del modelo, ahora considerados aceptables $\left(\chi^{2}=9.667[\mathrm{gl}=4] ; \mathrm{p}>0.05 ; \chi^{2} / \mathrm{gl}=2.416\right.$; CFI $=0.994 ;$ RMSEA $=0.053$ [IC $90 \%: 0.006-0.097$ ]; SRMR $=0.018$ ). Además, el AIC es muy inferior al primer modelo (tabla 1).

Tabla 1. Índices de bondad de ajuste estadístico de dos modelos de la Escala de Bienestar ( $\mathrm{n}=499)$

\begin{tabular}{cccccccc}
\hline & $\begin{array}{c}\chi^{2} \\
(\mathbf{g l})\end{array}$ & $\mathbf{p}$ & $\chi^{2} / \mathbf{g l}$ & SRMR & CFI & AIC & $\begin{array}{c}\text { RMSEA } \\
\text { [IC 90\%] }\end{array}$ \\
\hline Modelo 1 & $36.022(5)$ & 0.000 & 7.204 & 0.032 & 0.966 & 26.564 & $0.112[0.079,0.147]$ \\
Modelo 2 e1-e4 & $9.667(4)$ & 0.099 & 2.416 & 0.018 & 0.994 & 1.667 & $0.053[0.006,0.097]$ \\
\hline
\end{tabular}

$X^{2}$ : chi cuadrado; gl = grados de libertad; SRMR: raíz residual estandarizada cuadrática media; RMSEA: error cuadrático medio de aproximación; cFi: índice de ajuste comparativo; AIc: criterio de Akaike; ic: intervalos de confianza.

Además, se reportan las cargas factoriales de los cinco ítems (tabla 2), donde el modelo 2 presenta altas cargas factoriales para la totalidad de ítems que oscilan entre 0.625 para el ítem 5 y 0.814 para el ítem 1.

Tabla 2. Saturaciones de los ítems en los diversos modelos de la Escala de Bienestar

\begin{tabular}{lcc}
\hline \multicolumn{1}{c}{ Ítems } & Modelo 1 & Modelo 2 \\
\hline 1. Me he sentido alegre y de buen ánimo & 0.763 & 0.814 \\
2. Me he sentido tranquilo(a) y relajado(a) & 0.751 & 0.733 \\
3. Me sentido activo(a) y con energía & 0.733 & 0.710 \\
4. Me he levantado sintiéndome bien y descansado & 0.693 & 0.752 \\
5. Mi vida diaria ha tenido cosas interesantes para mí & 0.642 & 0.625 \\
& & \\
$\omega$
\end{tabular}

w: coeficiente omega; *: omega corregido para errores correlacionados.

La tabla 2 presenta los resultados que sustentan la hipótesis 2, donde se espera una fiabilidad aceptable. Se presenta el cálculo del coeficiente omega para el modelo 1 ( $\omega=0.787$ ); mientras que en el caso del modelo 2 se efectuó una corrección de errores de correlaciones y presenta un coeficiente aceptable $\left(\omega_{\text {corregido }}=0.758\right)$.

La hipótesis 3, donde se propone que la estructura factorial es invariante entre hombres y mujeres se presentan a continuación. El M1 mostró excelentes valores de ajuste. La M2 presentó índices CFI = 0.993 y RMSEA $=0.046(0.000 ; 0.086)$, así como un valor de $\mathrm{CFI}(\Delta \mathrm{CFI}<0.01)$ similar a M1. Estos resultados sugieren que el modelo base (M1) y el modelo con restricciones en las cargas (M2) son equivalentes. Posteriormente, se examinó la equivalencia entre interceptos (M3). donde los valores de ajuste fueron similares al M2: $\mathrm{CFI}=0.995, \mathrm{sRMR}=0.033 \mathrm{y}$ 
RMSEA $=0.033$ (0.000; 0.071). Asimismo, se observa que la diferencia entre M3 y M2 no excede el criterio requerido ( $\Delta$ сғг $<0.01$ ), y con ello se consigue una invarianza fuerte (tabla 3 ). Finalmente, se comparó la equivalencia entre los residuos (M4), donde los valores sugieren que la diferencia entre M4 y M2 es mínima, al estar en el rango esperado $(\Delta$ сFI $<0.01)$.

Tabla 3. Invarianza de medición de la Escala de Bienestar

\begin{tabular}{|c|c|c|c|c|c|c|c|c|}
\hline Modelo & $\chi^{2}$ (gl) & $\Delta \chi^{2}(\Delta \mathrm{gl})$ & $\begin{array}{c}\text { RMSEA } \\
\text { [Ic 90\%] }\end{array}$ & $\mathbf{p}$ & SRMR & CFI & $(\Delta \mathrm{cFI})$ & ( $\triangle$ RMSEA) \\
\hline General & $\begin{array}{r}9.667 \\
(4)\end{array}$ & - & $\begin{array}{r}0.053 \\
{[0.006,0.097]}\end{array}$ & 0.046 & 0.018 & 0.994 & - & \\
\hline Mujeres & $\begin{array}{r}4.961 \\
(4)\end{array}$ & - & $\begin{array}{r}0.014 \\
{[0.027,0.079]}\end{array}$ & 0.291 & 0.045 & 0.998 & - & 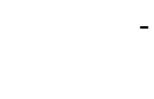 \\
\hline Hombres & $\begin{array}{r}10.047 \\
(4)\end{array}$ & - & $\begin{array}{r}0.081 \\
{[0.016,0.146]}\end{array}$ & 0.040 & 0.026 & 0.983 & - & \\
\hline M1 & $\begin{array}{r}15.008 \\
(8)\end{array}$ & - & $\begin{array}{r}0.059 \\
{[0.000,0.105]}\end{array}$ & 0.059 & 0.019 & 0.992 & - & \\
\hline M2 & $\begin{array}{r}18.362 \\
(12)\end{array}$ & $\begin{array}{r}3.354 \\
(4)\end{array}$ & $\begin{array}{r}0.046 \\
{[0.000,0.086]}\end{array}$ & 0.105 & 0.031 & 0.993 & 0.001 & 0.013 \\
\hline M3 & $\begin{array}{r}20.254 \\
(16)\end{array}$ & $\begin{array}{r}1.891 \\
(4)\end{array}$ & $\begin{array}{r}0.033 \\
{[0.000,0.071]}\end{array}$ & 0.209 & 0.033 & 0.995 & 0.002 & 0.013 \\
\hline M4 & $\begin{array}{r}21.894 \\
(21) \\
\end{array}$ & $\begin{array}{r}1.640 \\
(5) \\
\end{array}$ & $\begin{array}{r}0.013 \\
{[0.000,0.056]} \\
\end{array}$ & 0.406 & 0.037 & 0.999 & 0.004 & 0.020 \\
\hline
\end{tabular}

M1: configuracional; M2: métrica; M3: fuerte; M4: estricta; M5: medias latentes.

Una vez comprobada la invarianza factorial, se compararon las medias latentes, producto del cumplimiento de la invarianza fuerte (35). Los resultados demuestran que no existen diferencias estadísticamente significativas en el bienestar de acuerdo con el sexo $\left(t_{(494.97)}=\right.$ $-1.865 ; p=0.062)$. Asimismo, se calculó el tamaño del efecto $(d=-0.166)$ el mismo que resultó no existente (tabla 4$)$.

Tabla 4. Saturaciones de los ítems de acuerdo con el sexo

\begin{tabular}{lcc}
\hline \multicolumn{1}{c}{ Ítems } & גMujeres & $\lambda$ Varones \\
\hline 1. Me he sentido alegre y de buen ánimo & 0.809 & 0.824 \\
2. Me he sentido tranquilo(a) y relajado(a) & 0.756 & 0.699 \\
3. Me sentido activo(a) y con energía & 0.725 & 0.688 \\
4. Me he levantado sintiéndome bien y descansado. & 0.771 & 0.725 \\
5. Mi vida diaria ha tenido cosas interesantes para mí & 0.664 & 0.563 \\
\hline
\end{tabular}




\section{Discusión}

$\Lambda$ nte la ausencia de evidencias acerca de las propiedades psicométricas del wHo-5 wBI en -el Perú, este es el primer estudio cuyo objetivo fue analizar las evidencias de validez basada en la estructura interna, confiabilidad e invarianza factorial en función del sexo en población universitaria. Los resultados apoyan la estructura unidimensional, adecuada confiabilidad e invarianza factorial en función del sexo del wно-5 wвi, lo cual indica que es un instrumento adecuado para la evaluación del bienestar.

En términos de la evidencia de validez basada en el constructo, los resultados indican que la estructura unidimensional del wHo-5 wBI reportada en la literatura internacional se reproduce en la muestra de universitarios peruanos (18,21-24,26,39). Sin embargo, los hallazgos también apoyan la presencia de covarianzas de errores de los ítems 1 (Me he sentido alegre y de buen ánimo) y 4 (Me he levantado sintiéndome bien y descansado) en el modelo unidimensional. En este sentido, se sugiere la inclusión de este error correlacionado, debido a la similitud en el significado de ambos ítems (que podrían expresar el sentirse bien), debido a su modo de fraseo (especificidad) y no al error de medida. El componente específico que comparten los ítems 1 y 4 es que usan las palabras "sintiéndome bien” "y “buen ánimo”, que tienen un significado similar y que no comparten los otros tres ítems (40). Brown señala que la similitud conceptual y el fraseo son reconocidas como fuentes de varianza única (41). Este es uno de los pocos estudios en los cuales se presentan errores correlacionados, lo cual permite formular la hipótesis acerca de la influencia cultural y la heterogeneidad de la muestra en la estructura del wHo-5 wBi. Se recomienda que futuras investigaciones permitan la correlación entre los errores de los ítems 1 y 4. Ante estos resultados, se considera que una estructura de factor único modificada del who-5 wBI que permita correlacionar los errores de los ítems 1 y 4 es la más adecuada. La presencia de la correlación entre errores evitará a posteriori una inadecuada especificación de la estructura y una pérdida de información (40).

La presencia de errores correlacionados en el modelo 2 sobrestimaría el valor de la consistencia interna y dificultaría estimar sin sesgo el coeficiente alfa de Cronbach (42). Esto podría generar sesgos en el funcionamiento de los índices de ajuste (43). Ante esto, se utilizó el $\omega_{\text {corregido }}$ como estimador de la confiabilidad, debido a que su cálculo se basa en las cargas factorial y no depende del número de ítems ni del cumplimiento del principio de tal equivalencia (38). Así, se brinda evidencia empírica de confiabilidad satisfactoria, que es superior a lo reportado por Campo-Arias et al., pero inferior a lo señalado por SimancasPallares et al. $(19,25)$. Otros estudios han indicado mayores estimaciones de la confiabilidad de las puntuaciones; pero mediante el coeficiente alfa de Cronbach $(18,24,39,44,45)$.

Respecto a la invarianza factorial, los resultados señalan que la estructura del wHo-5 wBI mide el mismo constructo y es igual de precisa para universitarios de ambos sexos (35). Esto es consistente con los resultados de otros estudios que examinaron la invariancia factorial 
del who-5 wвi a través del sexo en Europa (23). De manera específica, la invariancia factorial sugiere que: 1) los universitarios de ambos sexos comprenden el bienestar en una misma estructura unidimensional (invarianza configural); 2) las asociaciones entre los ítems del wHO-5 wBI y el constructo latente (bienestar) no varía entre los grupos comparados. Esto sugiere que los universitarios contestaron de manera similar a todos los ítems, independientemente del sexo al que pertenezcan (invarianza métrica); 3) la relación entre el puntaje observado y el puntaje latente del who-5 wBI es invariante. De esta manera, un cambio en los puntajes latentes generaría el mismo cambio en los puntajes observados del wHo-5 wBI en los grupos de hombres y mujeres (invariancia escalar), y 4) el error de medición para cada ítem fue constante al comparar muestras de universitarios de ambos sexos (invarianza estricta).

Respecto a la comparación de medias latentes, no existen diferencias en el bienestar de acuerdo con el sexo. Este resultado es diferente a otro en el cual las mujeres presentan un mayor bienestar que los hombres, independientemente de la pregunta sobre bienestar que se utiliza, excepto en los países más pobres (46). Si bien este hallazgo es importante, la literatura sugiere investigar el rol mediador de la edad en la relación entre el sexo y bienestar (47).

La implicación directa de la invarianza factorial del wHO-5 wBI y el análisis de las diferencias de las medias latentes es que se brindará mayor solidez a las conclusiones derivadas de las comparaciones entre hombres y mujeres, justificándose el empleo del wHo-5 wBI en estudios comparativos por sexo. Además, estos resultados se consideran una evidencia adicional de la validez y fiabilidad del wHo-5 wBI.

Hasta donde se conoce de la literatura, este es el primer estudio que brinda evidencia de la falta de sesgo de medida del wHo-5 wBI entre hombres y mujeres en el contexto peruano y latinoamericano.

El estudio tiene algunas limitaciones. Primera, solo se llevó a cabo en universidades de Trujillo y utilizando un muestreo intencional no aleatorio. Esto limita la generalización de los hallazgos, por lo que se recomienda evaluar las propiedades psicométricas del wHO-5 WBI en universitarios de diferentes contextos culturales del país. Segunda, se evaluó en el periodo de ausencia de evaluaciones académicas parciales o finales, cuando es menor el estrés. La ausencia de estrés podría influir en el puntaje del segundo ítem que pregunta por "Me he sentido tranquilo(a) y relajado(a)". Tercera, los datos se obtuvieron a partir de una medida de autoinforme que puede resultar en un sesgo, debido a la deseabilidad social. Estudios futuros tienen que emplear evaluaciones sin autoinforme y muestras más representativas. Cuarta, solo se analizó la validez basada en la estructura interna; por lo que se recomienda el estudio futuro de la validez basada en el proceso de respuesta (consiste en analizar la congruencia entre el constructo y la naturaleza de la respuesta emitida por los examinados, a partir de entrevistas a profundidad u otro procedimientos similares), en el contenido (se trata de demostrar que los ítems del test son una muestra representativa del constructo que se pretende medir), 
en las consecuencias (referido al análisis de las consecuencias sobre el uso y las interpretaciones que se realizarán con el instrumento) y en la relación con otras variables (análisis de las puntuaciones del test con variables externas) con el objetivo de brindar mayor fuerza a las conclusiones. Quinta, no se evaluó la confiabilidad test-retest del wHo-5 wBI, lo que impide analizar la estabilidad de las puntuaciones a través del tiempo.

A pesar de las limitaciones, los resultados tienen importantes implicancias prácticas. Así, el confirmar la estructura unidimensional con la presencia de un error correlacionado, confiabilidad de las puntuaciones e invarianza factorial, evitará la presencia de sesgos en la medición del bienestar que puedan llevar a interpretaciones equivocadas de los resultados y que no estén asociadas con las características de los grupos evaluados. En este sentido, la estructura unidimensional se muestra sólida y robusta a las diferencias existentes entre hombres y mujeres, lo cual demuestra que el constructo es comprendido de forma similar por ambos subgrupos. De esta manera, se justifica el empleo del wHo-5 wBI, como medida breve y general que permita interpretaciones válidas y confiables del bienestar, dentro del modelo de universidades saludables, donde el profesional de ciencias de la salud cumple un papel importante (48). En este sentido, la evidencia señala que elevados niveles de bienestar son factores protectores ante el estrés y malestar emocional generados por los retos de la vida universitaria, al favorecer la calidad de vida, así como la salud física y mental (49).

En conclusión, el wно-5 कві es un instrumento breve, válido, confiable e invariante según sexo para la medición del bienestar en una muestra no clínica, como los estudiantes universitarios.

\section{Contribución de los autores}

Tomás Caycho-Rodríguez participó en la concepción y diseño, análisis e interpretación de 1 los datos, planeación del artículo y aprobación final de la versión para publicar.

José Ventura-León participó en el análisis e interpretación de los datos y aprobación final de la versión para publicar.

Karla Azabache-Alvarado participó en la revisión de contenido intelectual y aprobación final de la versión para publicar.

Mario Reyes-Bossio participó en la adquisición de los datos e información, revisión de contenido intelectual y aprobación final de la versión para publicar.

Isabel Cabrera-Orosco participó en la concepción y diseño, revisión de contenido intelectual y aprobación final de la versión para publicar. 


\section{Conflicto de intereses}

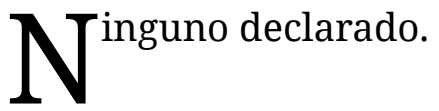

\section{Referencias}

1. Diener E, Oishi S, Tay L. Advances in subjective well-being research. Nat Hum Behav. 2018;2(4):253-60. https://doi.org/10.1038/s41562-018-0307-6

2. World Health Organization. Mental health action plan 2013-2020 [internet]. 2013. Disponible en: http://apps.who.int/iris/bitstream/10665/89966/1/9789241506021_eng.pdf

3. Diener E, Oishi S, Ryan KL. Universals and cultural differences in the causes and structure of happiness: a multilevel review. En Keyes C, editor. Mental well-being Dordrecht: Springer; 2013. p. 153-76.

4. Diener E, Oishi S, Lucas RE. National accounts of subjective well-being. Am Psychol. 2015;70(3):234-42. https://doi.org/10.1037/a0038899

5. Altun I, Özbağ GK, Arli E. Subjective Well-Being in University Students. Iran J Public Health. 2014;43(11):1585-6.

6. Rodríguez YR, Berríos AQ. El bienestar psicológico en el proceso de ayuda con estudiantes universitarios. Rev Griot. 2012;5(1):7-17.

7. Bailey TH, Phillips LJ. The influence of motivation and adaptation on students' subjective well-being, meaning in life and academic performance. High Educ Res Dev. 2016;35(2):201-16. https://doi.org/10.1080/07294360.2015.1087474

8. Gómez V, De Posada CV, Barrera F, Cruz JE. Factores predictores de bienestar subjetivo en una muestra colombiana. Rev Latinoam Psicol. 2007;39(2):311-25.

9. Hutz CS, Midgett A, Pacico JC, Bastianello MR, Zanon C. The relationship of hope, optimism, self-esteem, subjective well-being, and personality in Brazilians and Americans. Psychology. 2014;5(6):514-22. https://doi.org/10.4236/psych.2014.56061

10. Salmela-Aro K, Nurmi JE. Self-esteem during university studies predicts career characteristics 10 years later. J Vocat Behav. 2007;70(3):463-77. https://doi.org/10.1016/j. jvb.2007.01.006

11. Rania N, Siri A, Bagnasco A, Aleo G, Sasso L. Academic climate, well-being and academic performance in a university degree course. J Nurs Manag. 2014;22(6):751-60. https://doi. org/10.1111/j.1365-2834.2012.01471.x

12. Musikanski L. Measuring happiness to guide public policy making: a survey of instruments and policy initiatives. J Soc. Change. 2015;7(1):39-55. https://doi.org/10.5590/ JOSC.2015.07.1.04

13. Canadian Index of Wellbeing. How are Canadians really doing? The 2012 CIW Report. Waterloo, ON: Canadian Index of Wellbeing and University of Waterloo; 2012. 
14. Pontin E, Schwannauer M, Tai S, Kinderman P. A uk validation of a general measure of subjective well-being: the modified ввс subjective well-being scale (ввс-sшв). Health Qual Life Outcomes. 2013;11(1):150. https://doi.org/10.1186/1477-7525-11-150

15. World Health Organization. Well-being measures in primary health care: The DepCare Project. Consensus meeting, Stockholm: World Health Organization; 1998.

16. Topp CW, Østergaard SD, Søndergaard S, Bech P. The wHo-5 Well-Being Index: a systematic review of the literature. Psychother Psychosom. 2015;84(3):167-76. https://doi. org/10.1159/000376585

17. Garland AF, Deyessa N, Desta M, Alem A, Zerihun T, Hall KG, et al. Use of the who's Perceived Well-Being Index (wHo-5) as an efficient and potentially valid screen for depression in a low income country. Fam Syst Health. 2018;36(2):148-58. https://doi. org/10.1037/fsh0000344

18. Morelatto de Souza C, Loayza MP. World Health Organization 5-item well-being index: validation of the Brazilian Portuguese version. Eur Arch Psychiatry Clin Neurosci. 2012;262(3):239-44. https://doi.org/10.1007/s00406-011-0255-X

19. Campo-Arias A, Miranda-Tapia GA, Cogollo Z, Herazo E. Reproducibilidad del Índice de Bienestar General (WHO-5 WBI) en estudiantes adolescentes. Salud Uninorte. 2015;31(1):18-24. https://doi.org/10.14482/sun.30.1.4309

20. Péntek M, Hajdu O, Rencz F, Beretzky Z, Brodszky V, Baji P, et al. Subjective expectations regarding ageing: a cross-sectional online population survey in Hungary. Eur J Health Econ. 2019;20(1):17-30. https://doi.org/10.1007/s10198-019-01059-w

21. Bonnín CM, Yatham LN, Michalak EE, Martínez-Arán A, Dhanoa T, Torres I, et al. Psychometric properties of the well-being index (wHo-5) Spanish version in a sample of euthymic patients with bipolar disorder. J Affect Disord. 2018;228: 153-9. https://doi. org/10.1016/j.jad.2017.12.006

22. Omani-Samani R, Maroufizadeh S, Almasi-Hashiani A, Sepidarkish M, Amini P. The who5 Well-Being Index: a validation study in people with Infertility. Iran J Public Health. 2019;48(11):2058-64.

23. Guðmundsdóttir HB, Ólason DP, Guðmundsdóttir DG, Sigurðsson JF. A psychometric evaluation of the Icelandic version of the who-5. Scand J Psychol. 2014;55(6):567-72. https://doi.org/10.1111/sjop.12156

24. Mortazavi F, Mousavi SA, Chaman R, Khosravi A. Validation of the World Health Organization-5 Well-Being Index; assessment of maternal well-being and its associated factors. Turk Psikiyatri Derg. 2015;26(1):1-7.

25. Simancas-Pallares M, Díaz-Cárdenas S, Barbosa-Gómez P, Buendía-Vergara M, ArévaloTovar L. Propiedades psicométricas del Índice de Bienestar General-5 de la Organización Mundial de la Salud en pacientes parcialmente edéntulos. Rev Fac Med. 2016;64(4):7015. https://doi.org/10.15446/revfacmed.v64n4.52235

26. Krieger T, Zimmermann J, Huffziger S, Ubl B, Diener C, Kuehner C, Holtforth MG. Measuring depression with a well-being index: further evidence for the validity of the wно Well-Being Index (wHo-5) as a measure of the severity of depression. J Affect Disord. 2014;156: 240-4. https://doi.org/10.1016/j.jad.2013.12.015 
27. Abdulameer SA, Al-Jewari WM, Sahib MN. Psychological health status and salivary IgA among pharmacy students in Iraq: validation of pss-4 and wHo-5 well-being (Arabic version). Pharm Educ. 2019;19(1):10-8.

28. Preoteasa CT, Preoteasa E. Psychometric properties of Romanian version of who-5 well-being index in dental students. Rom J Oral Rehabil. 2015;7(3):21-7.

29. Byrne BM. Testing for multigroup equivalence of a measuring instrument: a walk through the process. Psicothema. 2008;20(4):872-2.

30. Ato M, López-García JJ, Benavente A. Un sistema de clasificación de los diseños de investigación en psicología. Anales de Psicología. 2013;29(3):1038-59. https://doi.org/10.6018/ analesps.29.3.178511

31. Schumacker R, Lomax R. A beginner's guide to structural equation modeling. New York: Routledge; 2016.

32. Mueller RO, Hancock GR. Best practices in structural equation modeling. En Osborne JW, editor. Best practices in quantitative methods. Thousand Oaks, ca: Sage; 2008. p. 488-508.

33. Hu LT, Bentler PM. Cutoff criteria for fit indexes in covariance structure analysis: Conventional criteria versus new alternatives. Struct Equ Modeling. 1999;6(1):1-55. https://doi.org/10.1080/10705519909540118

34. Whittaker TA. Using the modification index and standardized expected parameter change for model modification. J Exp Educ. 2012;80(1):26-44. https://doi.org/10.1080/0 0220973.2010 .531299

35. Dimitrov DM. Testing for factorial invariance in the context of construct validation. Meas Eval Couns Dev. 2010;43(2):121-49. https://doi.org/10.1177/0748175610373459

36. Cohen J. A power primer. Psychol Bull. 1992;112(1):155-9. https://doi.org/10.1037/00332909.112.1.155

37. McDonald RP. Test theory: A unified treatment. Mahwah, NJ: Erlbaum; 1999

38. Raykov T. Bias of coefficient afor fixed congeneric measures with correlated errors. Appl Psych Meas. 2001;25(1), 69-76. https://doi.org/10.1177/01466216010251005

39. Chongwo E, Ssewanyana D, Nasambu C, Mwangala PN, Mwangi PM, Nyongesa MK, Newton ChR, Abubakar A. Validation of a Swahili version of the World Health Organization 5-item well-being index among adults living with HIv and epilepsy in rural coastal Kenya. Glob Health Res Policy. 2018;3(1):26. https://doi.org/10.1186/s41256-018-0081-z

40. Saris WE, Satorra A, Sörbom D. The detection and correction of specification errors in structural equation models. Sociol Methodol. 1987;17: 105-29. https://doi. org/10.2307/271030

41. Brown TA. Confirmatory factor analysis for applied research. New York: Guilford Press; 2006.

42. Gu F, Little TD, Kingston NM. Misestimation of reliability using coefficient alpha and structural equation modeling when assumptions of tau-equivalence and uncorrelated errors are violated. Methodology. 2013;9(1):30-40. https://doi.org/10.1027/1614-2241/ a000052 
43. Heene M, Hilbert S, Freudenthaler HH, Bühner M. Sensitivity of SEM fit indexes with respect to violations of uncorrelated errors. Struct Equ Modeling. 2012;19(1):36-50. https://doi.org/10.1080/10705511.2012.634710

44. Dadfar M, Momeni Safarabad N, Asgharnejad Farid AA, Nemati Shirzy M, Ghazie pour Abarghouie F. Reliability, validity, and factorial structure of the World Health Organization-5 Well-Being Index (wHo-5) in Iranian psychiatric outpatients. Trends Psychiatry Psychother. 2018;40(2):79-84.

45. Rauwerda NL, Tovote KA, Peeters ACTM, Sanderman R, Emmelkamp PMG, Schroevers MJ, Fleer J. wHо-5 and BDI-II are acceptable screening instruments for depression in people with diabetes. Diabet Med. 2018;35(12), 1678-85. https://doi.org/10.1111/dme.13779

46. Graham C, Chattopadhyay S. Gender and well-being around the world. Int J of Happiness Dev. 2013;1(2):212-32.

47. Helliwell J, Layard R, Sachs J. Setting the stage. En Helliwell J, Layard R, Sachs J. editores. World happiness report 2015. New York: Sustainable Development Solutions Network; 2015. p. 3-11.

48. Becerra S. Universidades saludables: una apuesta a una formación integral del estudiante. Rev Psicol. 2013;31(2):287-314.

49. Barrantes-Brais K, Ureña-Bonilla P. Bienestar psicológico y bienestar subjetivo en estudiantes universitarios costarricenses. Rev Intercont Psicol Educ. 2015;17(1):101-23. 